\title{
ESTUDO COMPARATIVO, RANDOMIZADO DO USO DE DRENO ASPIRATIVO EM DERMOLIPECTOMIA ABDOMINAL
}

\section{COMPARATIVE, RANDOMIZED STUDY OF THE USE OF DRAINS IN ABDOMINAL DERMOLIPECTOMY}

\author{
Giuliano Borile'; ${ }^{1}$ Denis Valente, ACBC-RS ${ }^{1}$; \\ Francisco Tostes ${ }^{2}$; Eduardo $\mathrm{Chem}^{3}$; Roberto Correa $\mathrm{Chem}^{4}$
}

\begin{abstract}
RESUMO: Objetivo: As coleções líquidas, como hematoma e seroma, destacam-se como as complicações pós-operatórias mais freqüentes na prática cirúrgica da dermolipectomia abdominal. O seroma tem incidência variável e apresenta-se relativamente tardio. O hematoma pode apresentar-se clinicamente mais precoce que o seroma, porém, freqüentemente, também é um achado tardio devido ao espaço que pode ocupar antes que possa ser clinicamente detectável. O objetivo deste estudo é traçar o perfil dos pacientes submetidos a este procedimento e avaliar se a utilização ou não de drenos na ferida pós-operatória tem influência na formação de coleções líquidas pós-operatórias. Método: Este ensaio clínico, prospectivo e randomizado, estudou dois grupos de pacientes submetidos à dermolipectomia abdominal (com e sem drenagem pós-operatória) no Serviço de Cirurgia Plástica do Complexo Hospitalar Santa Casa de Porto Alegre. Os resultados foram avaliados clinicamente e submetidos a análise estatística. Resultados: Durante o período de janeiro de 2003 a abril de 2004, 63 pacientes foram submetidas à dermolipectomia. Em 30 (Grupo 1) foi utilizado dreno tubular fechado com pressão negativa, na ferida operatória. Em 33 (Grupo 2) não foi realizada qualquer drenagem de ferida operatória. Ocorreu um caso de seroma no Grupo 1 e dois de seroma e um hematoma no Grupo 2, sem significância estatística. Das 63 pacientes estudadas, 58\% tiveram a primeira gestação entre 16 e 20 anos e $19 \%$ apresentaram múltiplas gestações. Conclusão: O perfil das pacientes submetidas à dermolipectomia abdominal é de primigestas precoces, multíparas, com emagrecimento recente. A formação de coleções líquidas não foi afetada significativamente pelo uso ou não de drenos (Rev. Col. Bras. Cir. 2005; 32(5): 325-327).
\end{abstract}

Descritores: Cirurgia; Retalhos cirúrgicos; Abdome; Drenagem.

\section{INTRODUÇÃO}

A dermolipectomia abdominal permanece entre as cirurgias de contorno corporal freqüentemente realizadas na atualidade. Seu objetivo primário pode ser estético (pós-emagrecimento) ou estar associado à correção de deformidades da parede abdominal (hérnias, eventrações). O número absoluto desta modalidade cirúrgica tem aumentado de acordo com a maior difusão da cirurgia bariátrica ${ }^{1}$. Apesar das diferentes táticas e técnicas empregadas, a dinâmica cirúrgica baseia-se no descolamento dermo-gorduroso e ressecções em maior ou menor grau ${ }^{2}$. As complicações pós-operatórias mais freqüentes são as coleções líquidas, entre as quais destacamos o hematoma e o seroma ${ }^{3}$.

O seroma tem incidência variável e etiologia relacionada à alteração da velocidade de fechamento dos capilares linfáticos e apresenta-se relativamente tardio. Este, apesar de uma evolução benigna, pode apresentar alterações como encapsulamento, infecção, drenagens espontâneas entre outras, vindo a comprometer o resultado esperado.

O hematoma pode apresentar-se mais precocemente, porém, frequientemente, também é um achado tardio devi- do ao espaço que pode ocupar antes que possa ser clinicamente detectável ${ }^{3,4}$.

Na atualidade, a maioria dos cirurgiões opta pela drenagem (fechada ou aberta) pós-dermolipectomia no intuito de diminuir a taxa de coleções como seromas e hematomas. Esta prática é contestada por outro grupo que advoga a não drenagem pós-operatória, optando por compressão externa e/ou uso de pontos entre o retalho dermo-gorduroso e a fáscia no intuito de minimizar o mesmo tipo de complicação. Além de não prevenir o seroma, o abscesso pode causar desconforto e diminuição da mobilidade do paciente ${ }^{1,3,5}$.

O objetivo deste trabalho é comparar o uso ou não da drenagem pós-operatória no surgimento de coleções fluidas no pós-operatório de dermolipectomia abdominal, caracterizando a população submetida a este procedimento.

\section{MÉTODO}

Este estudo consistiu de um ensaio clinico randomizado, com os seguintes critérios de inclusão e exclusão:

Critérios de inclusão:

1. Cirurgião-Geral, Residente em Cirurgia Plástica do CHSC

2. Cirurgião Geral, Instrutor de Cirurgia Plástica do CHSC.

3. Cirurgião Geral, Instrutor de Cirurgia Plástica do CHSC, Mestre em Medicina.

4. Professor de Cirurgia Plástica da FFFCMPA, Chefe do Serviço de Cirurgia Plástica do CHSC, Mestre e Doutor em Medicina

Recebido em 24/08/2005

Aceito para publicação em 31/10/2005

Conflito de interesse : nenhum

Fonte de financiamento: nenhuma

Trabalho realizado no Serviço de Cirurgia Plástica do Complexo Hospitalar Santa Casa (CHSC) de Porto Alegre, RS. 
1. Receber atendimento no ambulatório do Serviço de Cirurgia Plástica do CHSC

2. Ser submetido à avaliação de grupo médico em regime de triagem para a indicação de abdominoplastia.

3. Ser portadora de lipodistrofia em abdômen inferior.

4. Ter índice de massa corporal entre 20 e $29 \mathrm{Kg} / \mathrm{m}^{2}$.

5. Assinar termo de consentimento informado.

Critérios de exclusão:

1. História de dermolipectomia prévia.

2. Expectativas mágicas em relação ao resultado operatório.

3. Doença psiquiátrica.

4. Discrasias sanguíneas.

5. Idade inferior a 18 ou superior a 70 anos.

No período de 01 de janeiro de 2003 a 20 de Abril de 2004 foram realizadas 63 dermolipectomias abdominais no serviço de Cirurgia Plástica da CHSC-FFFCMPA. Os pacientes, após a avaliação e indicação formal de dermolipectomia abdominal, foram alocados randomicamente em dois grupos. Ambos os grupos foram submetidos à cirurgia convencional. Para os paciente do grupo $1(\mathrm{n}=30)$ foi instituída a drenagem tubular fechada com pressão negativa até que a drenagem diária ficasse em torno de 30-50ml. Os pacientes do grupo $2(n=33)$ não receberam qualquer tipo de drenagem, permanecendo, apenas, com malha compressiva no pós-operatório. Ambos os grupos foram seguidos, após a alta hospitalar, sendo examinados semanalmente nas primeiras seis semanas. A partir de então, até o $3^{\circ}$ mês a cada 15 dias. Do terceiro ao sexto mês, os pacientes foram examinados mensalmente. Pacientes que apresentassem sinais clínicos sugestivos de coleção líquida como abaulamentos, deformidades de contorno, equimoses extensas ou ondas líquidas eram submetidos á punção com agulha e medição do volume aspirado.

Todos os casos foram avaliados segundo protocolo de pesquisa. Para análise estatística foi utilizado o teste de qui-quadrado e o teste exato de Fisher, considerando-se estatisticamente significativo valores de $p$ inferiores a 0,05 .

\section{RESULTADOS}

A Tabela 1 demonstra que ambos os grupos são comparáveis do ponto de vista estatístico em relação ao sexo, peso e altura . A idade e a técnica operatória também foram semelhantes em ambos os grupos.

Em relação à gravidez $58 \%$ apresentou a primeira gestação entre os 16 e 20 anos de idade e $19 \%$ apresentou pelo menos uma gestação múltipla. $\mathrm{O}$ número médio de gestações foi de 2,3 por paciente.

As queixas mais comuns foram em ordem decrescente: flacidez e excesso de pele abdominal, estrias supra e infraumbilicais, diastase de músculos retos abdominais e coxim adiposo.

Complicações menores como epidermólise e pequenas deiscências localizadas estiveram presentes em $19 \%$ dos
Tabela 1 - Comparação das Amostras.

\begin{tabular}{lcc}
\hline & Grupo 1 $(\mathbf{n = 3 0})$ & Grupo 2 $(\mathbf{n = 3 3})$ \\
\hline Sexo & Feminino & Feminino \\
Peso* & $63,4 \mathrm{Kg}$ & $65,6 \mathrm{~kg}$ \\
Altura* & $1,61 \mathrm{~m}$ & $1,64 \mathrm{~m}$ \\
\hline
\end{tabular}

$* p>0,0$

Tabela 2 - Ocorrência de Coleções Líquidas.

\begin{tabular}{lcc}
\hline & Grupo 1 $(\mathbf{n}=\mathbf{3 0})$ & Grupo 2 $(\mathbf{n}=\mathbf{3 3})$ \\
\hline Hematoma* $^{*}$ & 0 & 1 \\
Seroma $^{*}$ & 1 & 2 \\
\hline${ }^{*}>0,05$ & &
\end{tabular}

casos. Não foram identificadas complicações maiores como fasceítes e necroses extensas do retalho. Uma paciente apresentou hematoma volumoso tardio, necessitando transfusão de 3UI de concentrado de hemácias. Três pacientes haviam sido submetidas à cirurgia bariátrica com emagrecimento superior a $60 \%$ do sobrepeso.

O grupo 1 apresentou um caso de coleção líquida (seroma) diagnosticado e drenado em torno do $10^{\circ}$ dia pósoperatório. O grupo 2 apresentou três casos de coleções líquidas (um hematoma e dois seromas) diagnosticados em no $7^{\circ}$ (hematoma) e $9^{\circ}$ (seroma) dias pós-operatórios. Não houve diferença estatística entre os grupos 1 e 2 em relação a formação de coleções líquidas no pós-operatório (Tabela 2).

\section{DISCUSSÃO}

Coleções fluidas no pós-operatório de abdominoplastia como o seroma e hematoma permanecem como uma preocupação freqüente. Esse tipo de evento tem se mantido constante nos últimos anos. $\mathrm{O}$ aumento do número de cirurgias bariátricas promoveu um novo seguimento de cirurgias reparadoras após grandes emagrecimentos e o acréscimo proporcional no número dessas complicações ${ }^{1,5}$.

O seroma é um fenômeno autolimitado, fisiológico e reacional após grandes descolamentos teciduais. A causa provável é a interrupção dos canais linfáticos e vasculares e a formação de espaço morto como resultante do grande descolamento tecidual. Esse processo parece ser auto-regulado pela quantidade de fluido gerado e sua tendência é resolver-se sem maiores intervenções ${ }^{3,6,7}$. De acordo com os resultados aqui apresentados, acreditamos que a compressão com malha elástica (cerca de $25 \mathrm{mmHg}$ ) nos primeiros 15 dias pode prevenir a formação de grandes coleções que tragam desconforto para o paciente ou evoluam com encapsulamento ou infecção. Devido à sua ocorrência mais tardia (em torno do $6^{\circ}$ $10^{\circ}$ dias), o uso de drenos de aspiração parece não contribuir para a prevenção do processo.

Acreditamos que, em relação ao hematoma, o dreno desempenhe um papel importante de monitorização do sangramento nas primeiras 24 horas. Hematomas mais tardios, 
como os identificados neste estudo, não foram prevenidos pelo uso do dreno que era retirado em torno do primeiro ou segundo dia de pós-operatório (quando apresentavam drenagem inferior à $50 \mathrm{ml} / 24 \mathrm{~h}$ ). E a permanência de drenos por períodos prolongados não é recomendada pelo desconforto e diminuição da mobilidade do paciente, contribuindo para o aumento de morbidades como trombo-embolismo e infecções locais ${ }^{8,9}$.

Coleções fluidas têm sua taxa aumentada proporcionalmente ao descolamento realizado, e a espessura do panículo adiposo. Estas ocorrem com maior frequiência no abdômen do que em qualquer outro sítio anatômico ${ }^{10,11}$. Os casos de cole- ções líquidas aqui relatados estavam relacionados a emagrecimentos maiores de $10 \mathrm{~kg}$ com um amplo descolamento, até os arcos costais. Contornos irregulares que não foram observados durante a cirurgia, equimoses localizadas, ondas líquidas palpáveis ou visíveis ou coleções que mudam de posição com a movimentação do paciente implicam em acúmulo fluido e necessitam avaliação complementar e tratamento.

Conclui-se que o perfil da população deste estudo é na sua maioria composta de mulheres jovens, primigestas precoces, multíparas, com emagrecimento recente e que a formação de coleções líquidas não foi afetada significativamente pelo uso ou não de drenos.

\begin{abstract}
Background: Fluid collections such as hematoma and seroma are by far the most frequent complication in abdominal dermolipectomy. Seroma has variable incidence and late presentation. Hematoma can be an early finding but can be later too because of the dead space that can fill before it can be clinically detected. The objective of this study is to show the patient profile and to evaluate if drainage contribute in fluid collections formation. Methods: This Clinical Trial randomized 2 groups (with and without post-operative drains) that underwent to abdominal dermolipectomy at Plastic Surgery Service of the Santa Casa Hospital of Porto Alegre. Results: From January 2003 to April 2004, 63 patients underwent abdominal dermolipectomy. In 30 (Group 1) a closed drainage was used, and in 33 (Group 2) surgical wound was not drained. Seroma ocurred in one patient of Group 1 and in two of Group 2, and hematoma ocurred in one patient of Group 2, with no statistical significance. Of the 63 patients, 58\% had their first pregnancy between 16 and 20 years of age, and 19 had multiple pregnancies. Conclusions: We concluded that the patient profile is women, with early first pregnancy, multiple pregnancies and recent weight loose. The fluid collections were not affected by the use of post-operative drainage.
\end{abstract}

Key words: Surgery; Surgical flaps; Abdomen; Drainage.

\section{REFERÊNCIAS}

1. Kargi E, Akduman D, Dokuzoguz B, et al. Late complication of abdominoplasty in an obese patient: systemic inflammatory response syndrome and seroma. Plast Reconstr Surg. 2003;111(4):1568-71.

2. Baroudi R, Ferreira CA. Contouring the hip and the abdomen. Clin Plast Surg. 1996;23(4):551-72; discussion 572-3.

3. Hafezi F, Nouhi AH. Abdominoplasty and seroma. Ann Plast Surg. 2002;48(1):109-10.

4. Dillerud E. Abdominoplasty combined with suction lipoplasty: a study of complications, revisions, and risk factors in 487 cases. Ann Plast Surg. 1990;25(5):333-8; discussion 339-43.

5. Chaouat M, Levan P, Lalanne B, et al. Abdominal dermolipectomies: early postoperative complications and longterm unfavorable results. Plast Reconstr Surg. 2000;106(7):16148; discussion 1619-23.

6. Matarasso A. Minimal-access variations in abdominoplasty. Ann Plast Surg. 1995;34(3):255-63.

7. Dabb RW, Hall WW, Baroody M, et al. Circumferential suction lipectomy of the trunk with anterior rectus fascia plication through a periumbilical incision: an alternative to conventional abdominoplasty. Plast Reconstr Surg. 2004;113(2):727-32; discussion 733-4.
8. Manassa EH, Hertl CH, Olbrisch RR. Wound healing problems in smokers and nonsmokers after 132 abdominoplasties. Plast Reconstr Surg. 2003;111(6):2082-7; discussion 733-4.

9. Mohammad JA, Warnke PH, Stavraky W. Ultrasound in the diagnosis and management of fluid collection complications following abdominoplasty. Ann Plast Surg. 1998;41(5):498-502.

10. Scevola S, Youssef A, Kroll SS, et al. Drains and seromas in TRAM flap breast reconstruction. Ann Plast Surg. 2002;48(5):511-4.

11. Pollock H, Pollock T. Progressive tension sutures: a technique to reduce local complications in abdominoplasty. Plast Reconstr Surg. 2000;105(7):2583-6; discussion 2587-8.

Endereço para correspondência:

Giuliano Borile

Rua Marcelo Gama 530/203

90540- 040 - Porto Alegre - Rs

E-mail:afirma@brturbo.com

Tel. (51) 3343-6550 